\title{
Acute respiratory distress syndrome subphenotypes and therapy responsive traits among preclinical models: protocol for a systematic review and meta-analysis
}

Adrien Carla ${ }^{1}$, Bruno Pereira ${ }^{2}$, Hanifa Boukail ${ }^{1}$, Jules Audard ${ }^{1,3}$, Nathalie Pinol-Domenech ${ }^{4}$, Manuela De Carvalho ${ }^{4}$, Raiko Blondonnet ${ }^{1,3}$, Ruoyang Zhai ${ }^{3}$, Dominique Morand ${ }^{1}$, Céline Lambert ${ }^{2}$, Vincent Sapin ${ }^{3,5}$, Lorraine B. Ware ${ }^{6,7}$, Carolyn S. Calfee ${ }^{8}$, Julie A. Bastarache ${ }^{6,7,9}$, John G. Laffey ${ }^{10,11}$, Nicole P. Juffermans ${ }^{12}$, Lieuwe D. Bos ${ }^{12}$, Antonio Artigas ${ }^{13}$, Patricia R. M. Rocco ${ }^{14}$, Michael A. Matthay ${ }^{8}$, Daniel F. McAuley ${ }^{15}$, Jean-Michel Constantin ${ }^{16}$, Matthieu Jabaudon ${ }^{1,3,6^{*}}$ and for the ESICM Translational Biology Group of the Acute Respiratory Failure section

\begin{abstract}
Background: Subphenotypes were recently reported within clinical acute respiratory distress syndrome (ARDS), with distinct outcomes and therapeutic responses. Experimental models have long been used to mimic features of ARDS pathophysiology, but the presence of distinct subphenotypes among preclinical ARDS remains unknown. This review will investigate whether: 1) subphenotypes can be identified among preclinical ARDS models; 2) such subphenotypes can identify some responsive traits.

Methods: We will include comparative preclinical (in vivo and ex vivo) ARDS studies published between 2009 and 2019 in which pre-specified therapies were assessed (interleukin (IL)-10, IL-2, stem cells, beta-agonists,

corticosteroids, fibroblast growth factors, modulators of the receptor for advanced glycation end-products pathway, anticoagulants, and halogenated agents) and outcomes compared to a control condition. The primary outcome will be a composite of the four key features of preclinical ARDS as per the American Thoracic Society consensus conference (histologic evidence of lung injury, altered alveolar-capillary barrier, lung inflammatory response, and physiological dysfunction). Secondary outcomes will include the single components of the primary composite outcome, net alveolar fluid clearance, and death. MEDLINE, Embase, and Cochrane databases will be searched electronically and data from eligible studies will be extracted, pooled, and analyzed using random-effects models. Individual study reporting will be assessed according to the Animal Research: Reporting of In Vivo Experiments guidelines. Meta-regressions will be performed to identify subphenotypes prior to comparing outcomes across subphenotypes and treatment effects.

(Continued on next page)
\end{abstract}

\footnotetext{
* Correspondence: mjabaudon@chu-clermontferrand.fr

'Department of Perioperative Medicine, CHU Clermont-Ferrand,

Clermont-Ferrand, France

${ }^{3}$ GReD, CNRS UMR 6293, INSERM U1103, Université Clermont Auvergne,

Clermont-Ferrand, France

Full list of author information is available at the end of the article
}

\section{$\triangle B M C$}

(c) The Author(s). 2020 Open Access This article is licensed under a Creative Commons Attribution 4.0 International License, which permits use, sharing, adaptation, distribution and reproduction in any medium or format, as long as you give appropriate credit to the original author(s) and the source, provide a link to the Creative Commons licence, and indicate if changes were made. The images or other third party material in this article are included in the article's Creative Commons licence, unless indicated otherwise in a credit line to the material. If material is not included in the article's Creative Commons licence and your intended use is not permitted by statutory regulation or exceeds the permitted use, you will need to obtain permission directly from the copyright holder. To view a copy of this licence, visit http://creativecommons.org/licenses/by/4.0/ The Creative Commons Public Domain Dedication waiver (http://creativecommons.org/publicdomain/zero/1.0/) applies to the data made available in this article, unless otherwise stated in a credit line to the data. 
(Continued from previous page)

Discussion: This study will inform on the presence and underlying pathophysiological features of subphenotypes among preclinical models of ARDS and should help to determine whether sufficient evidence exists to perform preclinical trials of subphenotype-targeted therapies, prior to potential clinical translation.

Systematic review registration: PROSPERO (ID: CRD42019157236).

Keywords: Systematic review protocol, Acute respiratory distress syndrome, Preclinical, Subphenotypes

\section{Background}

The acute respiratory distress syndrome (ARDS) is a lifethreatening clinical syndrome of rapid-onset pulmonary failure characterized by a dysregulated inflammatory response that results in severe respiratory failure and the need for mechanical ventilation [1]. Although the prevalence of ARDS is low (1-5/10,000 inhabitants), its incidence is high in critically ill patients, as the syndrome is present in approximately $10 \%$ of patients upon admission to the intensive care unit (ICU) and in $24 \%$ of ICU patients under mechanical ventilation support [2]. Despite decades of research, mortality due to ARDS remains high, with significant long-term comorbidity and reduced quality of life in survivors [2]. Various pharmacotherapeutic agents have failed to improve ARDS outcomes and current treatment strategy is largely supportive, based on optimized ventilatory settings [1]. A key reason for the lack of specific pharmacological therapy is likely due to the heterogeneity within ARDS, which is diagnosed by a constellation of signs as defined by the Berlin criteria [3].

The clinical and biological heterogeneity within syndromes such as sepsis ARDS makes it essential to identify more homogeneous subphenotypes when investigating potential therapies $[4,5]$. There has been recent recognition of distinct subphenotypes (on the basis of clinical/biochemical variables, natural history, disease manifestation, and/or treatment response without any implication about mechanism) and endotypes (defined by a distinct functional/pathobiological mechanism) within patients with ARDS $[4,6,7]$. Therefore, while benefit has been demonstrated in numerous preclinical studies for many candidate therapies, most have failed to translate to improved patient outcomes in randomized clinical trials (RCTs), suggesting that the appropriate subset of patients to target with the novel therapies may not have been correctly identified [8].

The retrospective analysis of data from two large multicenter RCTs (the "ventilation with lower tidal volumes as compared with traditional tidal volumes for acute lung injury and the acute respiratory distress syndrome [ARMA] and "assessment of low tidal volume and elevated end-expiratory volume to obviate lung injury" [ALVEOLI] trials) [9] has first identified at least two ARDS subphenotypes within clinical ARDS which were differentiated by the presence of shock, metabolic acidosis and a higher inflammatory status (interleukin (IL)-6 and soluble tumor necrosis factor receptor (sTNFr)-1). These two phenotypes are sometimes referred to as "endotypes" because they seem defined by distinct degrees of inflammation, but their underlying mechanisms remain largely unknown. However, these subphenotypes were stable over the first 3 days of enrolment in both RCTs, suggesting that they are linked to true biological processes. The hyper-inflammatory subphenotype demonstrated significantly worse outcomes when compared to the hypo-inflammatory subphenotype, with higher mortality and lesser ventilator-free and organ failure-free days. The analysis of plasma biomarkers in patients with ARDS previously enrolled in the MARS prospective cohort also identified two biological subphenotypes, which were very similar to those found earlier and were again associated with distinct mortality rates [10]. In the ALVEOLI trial, where a lower positive end-expiratory pressure (PEEP) strategy was compared to a higher PEEP strategy, the two subphenotypes demonstrated a distinct response in terms of major outcomes, suggesting the potential for using this subphenotypic classification to identify therapy responsive traits. This hypothesis was further confirmed through secondary analysis of the fluids and catheters treatment trial (FACTT) in which the two inflammatory subphenotypes had distinct effects of the randomly assigned fluid management strategy on 90-day mortality [11]. The potential for the inflammation-based subphenotypic classification was again demonstrated in a secondary analysis of the hydroxymethylglutaryl-CoA reductase inhibition with simvastatin in acute lung injury to reduce pulmonary dysfunction-2 (HARP-2) trial, a multicenter trial investigating the potential of simvastatin as an anti-inflammatory therapy for ARDS. Subphenotyping based on the hypo- versus hyper-inflammatory profile identified a therapy response trait as evidenced by the improved survival of patients classified as hyperinflammatory and randomized to simvastatin [12].

Translation of basic research findings to clinical practice remains daunting because of the heterogeneity and complexity of ARDS [13]. A substantial number of preclinical models of experimental lung injury have been used to investigate mechanisms of lung injury and test novel therapies [14-16]. Although none of these experimental models fully reproduces all features of human lung injury, most of them are based on the reproduction, 
in animals, of known risk factors for ARDS, such as pulmonary or extrapulmonary sepsis, lipid embolism such as when secondary to bone fracture, and acid aspiration, among other clinical risks [14, 15, 17]. Recently, more translational models of acute lung injury have been used, such as the ex vivo human lung preparation [18-21]. Although the nature of the insult leading to acute lung injury in preclinical models grossly influences one or more features of human ARDS, the presence of subphenotypes among experimental models of ARDS has never been systematically assessed to date.

\section{Study question}

We hypothesize that ARDS subphenotypes may exist among preclinical ARDS studies and that they may have distinct treatment response to select therapies.

To test this hypothesis, we designed a systematic review and meta-analysis of controlled preclinical trials in experimental, - in vivo (animal) and ex vivo (human lung preparation) -, models of ARDS. The primary goal of the review is to compare the key features of preclinical ARDS, as defined by the American Thoracic Society (ATS) consensus conference (histologic evidence of lung injury, altered alveolar-capillary barrier, lung inflammatory response, and physiological dysfunction) [15], in lung-injured experimental groups treated or not with any of some prespecified candidate therapies.

\section{Methods}

\section{Protocol and registration}

The systematic review and meta-analysis protocol originated from discussions between our team of translational research scientists (AC, JA, RB, DM, VS, JMC, MJ), information system specialists (NPD, MDC), a statistical and methodologist (BP), and the European Society of Intensive Care Medicine (ESICM) Translational Biology Group of the Acute Respiratory Failure section. The protocol was registered on PROSPERO (ID: CRD42019157236) in December 2019.

\section{Study eligibility criteria}

We will include controlled comparative studies (randomized and non-randomized) of preclinical acute lung injury/ARDS published from 2009 to 2019 that evaluate the efficacy and safety outcomes of a number of predefined therapies (stem cells, fibroblast growth factors, IL2, IL-10, beta-agonists, corticosteroids, modulators of the receptor for advanced glycation end-products (RAGE) pathway, anticoagulants, and halogenated agents). In order to collect sufficient data to test our hypotheses, it was decided a priori to include only studies in which at least two features, out of the four key features of preclinical ARDS proposed by the 2011 Official ATS Workshop Report [15], are assessed.
Table 1 Preclinical models of acute respiratory distress syndrome included in the review

\author{
Oleic acid \\ Lipopolysaccharides \\ Acid aspiration \\ Hyperoxia \\ Saline lavage \\ Pulmonary ischemia/reperfusion \\ Nonpulmonary ischemia/reperfusion \\ Intravenous bacteria \\ Intrapulmonary bacteria \\ Peritonitis \\ Cecal ligation and puncture \\ Trauma/Shock \\ Mechanical ventilation (ventilator-induced lung injury) \\ Chemical or chemotherapeutic injury (such as detergents) \\ Pancreatitis \\ Burns
}

\section{Preclinical model eligibility criteria}

We will include preclinical in vivo animal and ex vivo human models of experimentally induced acute lung injury that mimic at least some aspects of the pathophysiology of humans with ARDS according to the ATS consensus criteria [15]. The inclusion of a wide range of acute lung injury models should enhance the generalizability of our study findings. Acute lung injury in experimental models may be induced by several methods (Table 1). These include intravenous, intratracheal or intraperitoneal administration of bacteria or endotoxin, induction of injury by the ventilator (ventilator-induced lung injury), chemical agents (oleic acid, hydrochloric acid), trauma, shock (for example hemorrhagic or induced by sepsis), or remote organ injury (for example pancreatitis, ischemiareperfusion). We will exclude neonatal animal models of acute lung injury because the mechanisms of disease, and efficacy of treatments, are likely to be different in this specific setting.

\section{Interventions}

Preclinical intervention groups will include animals and ex vivo human lungs from studies that examine the therapeutic use of stem cells (and their derived microvesicles), fibroblast growth factors, IL-2, IL-10, betaagonists, corticosteroids, RAGE modulators, anticoagulants, and halogenated agents such as isoflurane, sevoflurane, and desflurane (Table 2). These interventions have been selected a priori because they are the main areas of interest of our research groups [22-30]. In addition to studies of therapies that are administered after lung injury is modeled, experiments using 
Table 2 Predefined list of select therapies included in the meta-analysis

\begin{tabular}{|c|c|}
\hline Class & Example of therapies \\
\hline Interleukins & IL-10, IL-2 \\
\hline Cell therapy & MSCs, MSC-derived microvesicles \\
\hline Adrenergic beta-agonists & Salbutamol, albuterol, sultanol, proventil \\
\hline Growing factors & Fibroblast growing factors, keratinocyte growth factor, endothelial growth factor \\
\hline RAGE modulators & Anti-RAGE antibody, recombinant soluble RAGE, gene knockout, endogenous secretory RAGE \\
\hline Corticosteroids & $\begin{array}{l}\text { Glucocorticoids, mineralocorticoids, 3-oxo steroid, 17-ketosteroids, domolene, cortifan, epicortisol, komed-hc, heb-cort, } \\
\text { prednisone, encortone, panasol, deltacortisone, decortisyl, decortin, predeltin, orasone, prednidib, prednisone, dacortin, } \\
\text { cortancyl, solupred, deltasone, pronisone, sterapred, encorton, panafcort, delta-dome, dehydrocortisone, prednisonum, } \\
\text { lisacort, ultracorten, winpred, metacortandracin, meticorten, prednisolone, predate, prednisolone, di-adreson f, delta-1- } \\
\text { hydrocortisone, prednisolonum, sterane, meti derm, metacortandralone, predonine }\end{array}$ \\
\hline Anticoagulant agents & $\begin{array}{l}\text { Heparin, antithrombin, plasminogen activator, fibrinolytic agents, tissue factor pathway inhibitor, } \\
\text { lipoprotein-associated coagulation inhibitor, thrombin-thrombomodulin complex, thrombin receptor, activated protein } \\
\text { C, drotrecogin alfa activated }\end{array}$ \\
\hline Halogenated agents & Sevoflurane, desflurane, isoflurane \\
\hline
\end{tabular}

IL interleukin; MSCs mesenchymal stem (stromal) cells; RAGE receptor for advanced glycation end-products

pretreatment will also be included since they are clinically relevant for the prevention of ARDS. Moreover, studies using a co-treatment or multiple injurious hits will be included.

\section{Comparison}

The preclinical comparison group will include data from studies that have had experimental acute lung injury induced but have not been administered any of the prespecified therapies listed in the previous paragraph. This will allow to perform effect size calculations in our meta-analysis to examine how efficacious and safe these therapies are in preclinical ARDS in general, and in preclinical ARDS subphenotypes in particular. In addition, we will use other control groups, such as healthy or sham-injured controls, to examine the severity of preclinical ARDS.

\section{Preclinical primary endpoint}

The primary endpoint is a composite of the four key features that define preclinical ARDS, with specific measurements defined by the consensus criteria proposed by the ATS [15].

These include histological evidence of tissue injury (usually based on the lung injury score), alteration of the alveolar-capillary barrier (for example increased concentration of high molecular weight proteins in bronchoalveolar fluid), measures of the inflammatory response in the lung (for example pulmonary leukocytes or neutrophils, cytokines), and measures of physiological dysfunction such as the alveolar-arterial gradient of oxygen concentration and the partial pressure of arterial oxygen $\left(\mathrm{PaO}_{2}\right)$ to fraction of inspired oxygen $\left(\mathrm{FiO}_{2}\right)$ ratio. These quantitative features and measurements are summarized in Table 3. We will focus on the "very relevant" measurements defined by the consensus criteria [15].
Because the assessment of our composite endpoint must reflect that the timing of experimental induction of ARDS may vary across studies, the primary endpoint will be analyzed in categories of time from the induction of ARDS of less than $6 \mathrm{~h}$, between 6 and $24 \mathrm{~h}$, and greater than $24 \mathrm{~h}$. These time points for measurement were selected since the development of inflammation and acute lung injury occurs over time and contributes to death and morbidity in this population [2].

\section{Preclinical secondary endpoints}

Secondary endpoints will include death, net alveolar fluid clearance, and each individual component of the primary composite endpoint. The same categories of time will be used for primary and secondary endpoints. Where reported, the occurrence of adverse events will also be documented for each included study. For animal models that use infectious induction of acute lung injury, we will describe pathogen clearance (for example number of bacterial colony-forming units).

\section{Information sources}

Search strategies and equation of searching have been developed and tested with the help of two librarians using the Peer Review of Electronic Search Strategies (PRESS) template (see Additional file 1 for the representative search strategy) [31]. We will search on MEDLINE, Embase, and Cochrane databases. Search strategies will use a combination of controlled vocabulary (for example corticoids, mesenchymal stem cells, acute lung injury, respiratory distress syndrome) and keywords (for example mesenchymal stromal cells, acute lung injury, acute/adult respiratory distress syndrome). Vocabulary and syntax will be adjusted across the databases. Two recently published animal filters [32, 33], validated for PubMed/MEDLINE and Embase and 
Table 3 Acute lung injury features and measurements

\begin{tabular}{ll}
\hline Features & Relevant measurements \\
\hline Histological evidence of lung injury & Accumulation of neutrophils in the alveolar/interstitial space \\
& Formation of hyaline membranes \\
& Proteinaceous debris in alveolar space \\
& Proteinaceous debris in alveolar space \\
& Thickening of the alveolar wall \\
& Injury by a standardized histology score \\
& Evidence of hemorrhage \\
& Areas of atelectasis \\
& Gross macroscopic changes such as discoloration of the lungs \\
& Increased extravascular lung water content \\
& Accumulation of protein/tracer in airspaces/extravascular space \\
& Total BAL protein concentration \\
& BAL concentration of high molecular weight proteins \\
& Vascular filtration coefficient \\
& Translocation of a protein from the airspaces into plasma \\
& Increased lung lymph flow, lymph protein concentration \\
& BAL total neutrophil counts \\
& Lung MPO activity \\
Concentrations of cytokines and chemokines in lung tissue, in BAL fluid, and/or in plasma & Hypoxemia \\
Inflammation & Increased A-a oxygen difference
\end{tabular}

$B A L$ bronchoalveolar lavage; MPO myeloperoxidase

amended slightly, will be used to increase relevancy. These filters will be adjusted for use in the other databases where a validated filter is unavailable. Language will be restricted to English studies and no studies published before 2009 will be included. The bibliographies of included studies and relevant reviews will also be hand searched for further preclinical studies.

\section{Study selection}

The titles and abstracts of search results will be screened independently by two investigators (AC, MJ). The full text of all potentially eligible studies will be retrieved and reviewed for eligibility. Disagreements between reviewers will be resolved by consensus or by a third member of the systematic review team (JA). Reasons for exclusion of potentially eligible studies will be recorded to enable a transparent selection process and to be in accordance with the Preferred Reporting Items for Systematic Reviews and Meta-Analyses (PRISMA) guidelines developed for proper reporting of clinical systematic reviews [34]. The Preferred Reporting Items for Systematic Reviews and MetaAnalyses Protocols (PRISMA-P) checklist for this review protocol is available as Additional file 2.

\section{Data collection and process and data items}

Data will be extracted by four members (AC, JA, RZ, MJ) of the research team and collected into standardized, pilot-tested forms using REDCap electronic data capture tools hosted at CHU Clermont-Ferrand [35]. REDCap (Research Electronic Data Capture) is a secure, web-based application designed to support data capture for research studies, providing: (1) an intuitive interface for validated data entry; (2) audit trails for tracking data manipulation and export procedures; (3) automated export procedures for seamless data downloads to common statistical packages; and (4) procedures for importing data from external sources. Specific data elements are listed in Table 4.

\section{Assessment of risk of bias}

The risk of bias will be evaluated by two reviewers (AC, MJ) for each included preclinical study. Since no validated and standardized risk of bias checklist exists for preclinical studies, we will describe the biases of the included studies using the Cochrane risk of bias assessment [36] and the Risk Of Bias In Nonrandomized Studies - of Interventions (ROBINS-I) [37] tools. Items in this tool include assessments for concealment of allocation, random sequence generation, blinding of personnel and the endpoint measurements, and completeness of endpoint reporting. Each bias criterion will be assigned a value of low, high, or unclear risk of bias for each included study [36].

\section{Assessment of construct validity and external validity}

We will also record features that will facilitate judgements of construct validity and external validity [38]. Construct validity in preclinical research concerns the extent to which an experimental system accurately models a clinical entity. These will include: age, gender, weight, species, and strain of animal; presence of comorbidities; type of acute lung injury model; timing, dose and mode of administration of treatment; and use of cointerventions (for example fluid resuscitation, type of 
Table 4 Data collection elements

\begin{tabular}{ll}
\hline Categories & Main items \\
\hline Study characteristics & $\begin{array}{l}\text { DOI, Num ID (specific number ID for the purpose of the meta-analysis), title, author, year, country and } \\
\text { journal of publication, country of study, mono/multicentric }\end{array}$ \\
$\begin{array}{l}\text { Study population (experimental model) } \\
\text { Type of acute lung injury model }\end{array}$ & $\begin{array}{l}\text { Animal species or ex vivo model, age, gender, strain, weight, and comorbidity } \\
\text { Intervention }\end{array}$ \\
$\begin{array}{l}\text { Co-intervention } \\
\text { Preclinical endpoints }\end{array}$ & Type (see Table 2), number, timing, mode of administration, dose \\
$\begin{array}{l}\text { Risk of bias assessments (Cochrane risk of bias } \\
\text { tool) }\end{array}$ & Randomitation fluids, antibiotics, mechanical ventilation parameters, mode of anesthesia, drugs, etc. \\
$\begin{array}{l}\text { Quality of reporting of individual preclinical } \\
\text { studies }\end{array}$ & ARRIVE (Animal Research: Reporting of In Vivo Experiments) guidelines elements \\
\hline
\end{tabular}

DOI digital object identifier

anesthesia, use of antibiotics for infectious acute lung injury models) (Table 5). External validity in preclinical research concerns the extent to which cause and effect relationships holdup under varied conditions [39], such as the use of a multicenter preclinical design.

\section{Description of reporting}

We will describe the quality of reporting of the included preclinical studies using the elements of the Animal Research: Reporting of In Vivo Experiments (ARRIVE) guidelines. The ARRIVE guidelines were developed to enhance the transparent and comprehensive reporting of research methods and results for in vivo animal experiments [40].

\section{Ethics and dissemination}

This study does not require ethical approval. Data collected through this systematic review will be managed by the ESICM Translational Biology Group and made available upon reasonable request. The results of this systematic review will be presented at relevant conferences and published in a peer-reviewed journal.

\section{Data analysis}

Statistical analyses will be performed using Stata (version 15; StataCorp, College Station, US), Comprehensive Meta-Analysis (version 2; Biostat, Englewood, NJ), and Mplus [41] softwares. For descriptive analyses, data will be presented as mean and standard deviation or median and interquartile range, according to statistical distribution. To address the non-independence of data due to study effect, random-effects models will be performed rather than fixed-effect as certain experimental parameters can have wide variation. The dichotomous endpoints from each included study will be pooled and described as relative risks (RR) and 95\% confidence intervals incorporating a random effects modeling approach with the use of forest plots for presentation of the data [42]. Continuous endpoints will be pooled using the ratio of weighted means method with inverse variance random effects modeling and Hedges's

Table 5 Elements of construct validity and external validity

\begin{tabular}{|c|c|}
\hline Category & Items \\
\hline Animal species and strain (for mice) & Human lung preparation, mouse, rat, sheep, pig, rabbit, dog or others \\
\hline Animal age & Young, middle-aged, and mature, depending on species \\
\hline Animal gender & Male, female, both \\
\hline Comorbidity & Yes or no, type \\
\hline Model of acute lung injury & As defined in Table 1 \\
\hline Severity of lung injury & Lung injury score \\
\hline Intervention type & The drug, mode of administration and dose \\
\hline Timing of intervention regarding to acute lung injury induction & Before lung injury, 0 to 1,1 to 6 , and $>6 \mathrm{~h}$ after lung injury \\
\hline Type of control & Injured, non-injured \\
\hline Use of co-interventions & $\begin{array}{l}\text { Resuscitation fluids, use of antibiotics, type of anesthesia, mechanical ventilation } \\
\text { strategy }\end{array}$ \\
\hline Number of study centers & Single versus multi-center \\
\hline
\end{tabular}


standardized mean differences (SMD) [43]. When necessary, the RRs reported in individual studies will be converted into SMD [44]. The statistical heterogeneity of included preclinical studies will be measured using the $\mathrm{I}^{2}$ [45], which will be used to measure heterogeneity with 25,50 and $75 \%$ indicating low, moderate and high heterogeneity, respectively. Egger's test and funnel plots will be used to assess publication bias. In the absence of bias, studies will be distributed evenly around the mean effect size because of random sampling error.

Then, meta-regression will be conducted, with covariates determined according to the aforementioned analyses and to clinical and/or biological relevance, and methodological issues such as the categories of time from ARDS induction. Particular attention will be paid to the study of multicollinearity and interactions between covariates 1 ) studying the relationships between the covariates and 2) evaluating the impact of adding or removing variables to or from the multivariable model. Whenever possible in appropriate data, latent class analyses will be carried out to identify subphenotypes within experimental models of ARDS, associated with distinct outcomes and differential responses to therapy. Statistical analyses will be conducted including all controlled comparative studies, with the type of study considered as stratification variable (randomized and non-randomized).

When the number of studies and sample size will permit, analyses will be performed by study type. Several subgroup analyses to examine preclinical heterogeneity will be conducted on the primary endpoint. These analyses will include "clinical"/biological features (such as the type of experimental model; animal age, gender, weight, species, and strain; presence of comorbidities; cause of experimental ARDS; severity of the ARDS model; type of tested therapies (Table 2); type of controls; type of anesthesia; use of co-interventions, antibiotics, and mechanical ventilation settings; multiple-hit versus single-hit model of ARDS) and methodological features (such as single versus multi-center study, presence of an a priori sample size calculation). These subgroup analyses will be exploratory and the results will be interpreted with caution. In addition, subphenotypes that may be identified among preclinical studies will be compared to those already described in Humans (and those that could be identified in the future) using the same set of variables than in patients such as plasma levels of some proinflammatory cytokines, when applicable $[6,7]$.

Sensitivity analyses will be conducted to assess how including and excluding studies influences the results. More precisely, sensitivity analyses will be performed to measure the impact of high heterogeneity or a methodological quality estimated too low, and risk of bias assessments. In addition, sensitivity analyses will be performed, when possible, to assess the effects of the nature, sequence, and timing of all the injurious hits that are used in studies based on multiple-hit models of experimental ARDS.

\section{Discussion}

The results of this systematic review and meta-analysis will inform translational and clinical scientists, researchers, and clinicians internationally regarding the existing preclinical evidence for subphenotypes among preclinical, in vivo or ex vivo models of ARDS. Such data will be crucial to confirm the potential of personalized ARDS medicine $[6,8,46]$, i.e. the promise to deliver the right -targeted- treatment to the right subject. These data will also inform on the potential value of testing candidate therapies (including therapies that have already been tested in previous studies) that target distinct functional and/or pathobiological processes (such as lung epithelial injury and alveolar inflammation [9, 47]) in select experimental models that might best mimic ARDS subphenotypes described in patients to date [6]. For example, although it seems plausible that tracheal instillation with hydrochloric acid causes prominent injury to the alveolar epithelium and live bacteria (such as Streptococcus pneumoniae) causes major alveolar inflammation profiles, it remains unknown whether these models might have reliable value to represent clinical subphenotypes of higher inflammation and of higher degree of lung epithelial injury, respectively [6].

Our review is timely since there is an increasing amount of research dedicated to the identification of ARDS subphenotypes, their underlying mechanisms, and their potential responsive treats $[4,7,9-12,48-52]$. The results from this study should therefore be useful in order to generate hypotheses on whether some therapies (see Table 2) might differentially benefit to some preclinical subphenotypes, as compared to others. In case of positive results, this might open new perspectives on preclinical precision therapies, with the ultimate goal of future clinical translation. However, the predefined, limited number of therapies investigated through our review (see Table 2) is a limitation that should be acknowledged; this limited number reflects both our specific research topics of interest and a balance between scientific validity and feasibility. Our results will inform on whether the nature of the lung injurious hit used in published experimental models of ARDS could identify subphenotypes with distinct degrees of severity and treatment effects. Yet, we might not be able to distinguish such subphenotypes using individual subject data analysis in a given experimental model, such as recently reported in a secondary analysis of a murine cecal ligation and puncture sepsis model, with one subphenotype characterized by faster deterioration and more severe inflammation than the other and distinct responses 
to some interventions (immediate versus delayed antibiotics and fluids) [53].

In a broader perspective, we also hope this review will increase our knowledge on, and identify challenges and barriers related to, the conduct of preclinical ARDS studies. Ultimately, it will also inform and enrich future preclinical and clinical precision, subphenotype-based, research that should aid the translation of novel therapies for ARDS.

\section{Supplementary information}

Supplementary information accompanies this paper at https://doi.org/10. 1186/s12931-020-01337-9.

Additional file 1. Representative Search Strategy.

Additional file 2. Preferred reporting items for systematic review and meta-analysis protocols (PRISMA-P) checklist.

\section{Abbreviations}

ALVEOLI: Assessment of low tidal volume and elevated end-expiratory volume to obviate Lung injury; ARDS: Acute respiratory distress syndrome; ARMA: Ventilation with lower tidal volumes as compared with traditional tidal volumes for acute lung injury and the acute respiratory distress syndrome; ARRIVE: Animal research: reporting of in vivo experiments; ATS: American thoracic society; ESICM: European society of intensive care medicine; FACTT: Fluids and catheters treatment trial; $\mathrm{FiO}_{2}$ : Fraction of inspired oxygen; HARP-2: Hydroxymethylglutaryl-CoA reductase inhibition with simvastatin in acute lung injury to reduce pulmonary dysfunction-2; ICU: Intensive care unit; IL: Interleukin; $\mathrm{PaO}_{2}$ : Partial pressure of arterial oxygen; PEEP: Positive end-expiratory pressure; PRESS: Peer review of electronic search strategies; PRISMA: Preferred reporting items for systematic reviews and meta-analyses; PRISMA-P: Preferred reporting items for systematic reviews and meta-analyses protocols; REDCAP: Research electronic data capture; RAGE: Receptor for advanced glycation end-products; RCT: Randomized clinical trial; ROBINS-I: Risk of bias in nonrandomized studies - of interventions; RR: Relative risk; SMD: Standardized mean differences; sTNFr-1: Soluble tumor necrosis factor receptor 1

\section{Acknowledgements}

Not applicable

\section{Patient and public involvement (PPI) statement}

This research was done without patient involvement. Patients were not invited to comment on the study design and were not consulted to develop patient relevant outcomes or interpret the results. Patients were not invited to contribute to the writing or editing of this document for readability or accuracy.

\section{Authors' contributions}

AC, BP, DM, and MJ conceived the protocol design for this systematic review, and $\mathrm{MJ}$ is its guarantor. $\mathrm{AC}, \mathrm{BP}$, and $\mathrm{MJ}$ were responsible for initial drafting and manuscript revisions. AC, HB, NPD, MDC, RZ, JA, and MJ were responsible for the data collection strategy and preparation. $C L$ and $B P$ provided critical revisions and will be responsible for data management and statistical analysis. JMC, VS, LBW, CSC, JAB, JGL, NPJ, LDB, AA, PRMR, MAM, and DMA provided critical revisions in the design of the review protocol, shared their expertise in translational biology, and provided oversight for planned eligibility criteria and outcome measures. AC and MJ will oversee knowledge translation. All authors reviewed several drafts of the manuscript and approved the final version.

\section{Funding}

This research received no specific grant from any funding agency in the public, commercial or not-for-profit sectors.

\section{Availability of data and materials}

Data collected through this systematic review will be managed by the ESICM Translational Biology Group and made available upon reasonable request.

Ethics approval and consent to participate

Not applicable.

\section{Consent for publication}

Not applicable.

\section{Competing interests}

The authors declare that they have no competing interests.

\section{Author details}

${ }^{1}$ Department of Perioperative Medicine, CHU Clermont-Ferrand, Clermont-Ferrand, France. ${ }^{2}$ Biostatistics Unit, Department of Clinical Research and Innovation (DRCI), CHU Clermont-Ferrand, Clermont-Ferrand, France. ${ }^{3}$ GReD, CNRS UMR 6293, INSERM U1103, Université Clermont Auvergne, Clermont-Ferrand, France. ${ }^{4}$ Université Clermont Auvergne, Health Library, Clermont-Ferrand, France. ${ }^{5}$ Department of Medical Biochemistry and Molecular Biology, CHU Clermont-Ferrand, Clermont-Ferrand, France. ${ }^{6}$ Division of Allergy, Pulmonary, and Critical Care Medicine, Department of Medicine, Vanderbilt University Medical Center, Nashville, TN, USA. ${ }^{7}$ Department of Pathology, Microbiology, and Immunology, Vanderbilt University Medical Center, Nashville, TN, USA. ${ }^{8}$ Division of Pulmonary and Critical Care Medicine, Departments of Medicine and Anesthesia, Cardiovascular Research Institute, University of California San Francisco, San Francisco, CA, USA. 'Department of Cell and Developmental Biology, Vanderbilt University, Nashville, TN, USA. ${ }^{10}$ Keenan Research Centre for Biomedical Science, Hospital for Sick Children, Departments of Anesthesia and Critical Care Medicine, St. Michael's Hospital, Departments of Anesthesia, Physiology and Interdepartmental Division of Critical Care, University of Toronto, Toronto, Canada. ${ }^{11}$ Regenerative Medicine Institute at CÚRAM Centre for Research in Medical Devices, National University of Ireland Galway, Galway, Ireland. ${ }^{12}$ Department of Intensive Care Medicine, Department of Respiratory Medicine, and Laboratory of Experimental Intensive Care and Anesthesiology, Amsterdam University Medical Center, Amsterdam, The Netherlands. ${ }^{13}$ Corporació Sanitaria Parc Tauli, CIBER de Enfermedades Respiratorias, Autonomous University of Barcelona, Barcelona, Spain. ${ }^{14}$ Laboratory of Pulmonary Investigation, Carlos Chagas Filho Institute of Biophysics, Federal University of Rio de Janeiro, Rio de Janeiro, Brazil. ${ }^{15}$ Wellcome-Wolfson Institute for Experimental Medicine, Queens University Belfast and Regional Intensive Care Unit, Belfast Health and Social Care Trust, Belfast, UK. ${ }^{16}$ Department of Anesthesiology and Critical Care, Sorbonne University, GRC 29, AP-HP, DMU DREAM, Pitié-Salpêtrière Hospital, Paris, France.

Received: 23 January 2020 Accepted: 17 March 2020

Published online: 07 April 2020

\section{References}

1. Thompson BT, Chambers RC, Liu KD. Acute respiratory distress syndrome. N Engl J Med. 2017;377:562-72.

2. Bellani G, Laffey JG, Pham T, Fan E, Brochard L, Esteban A, et al. Epidemiology, patterns of care, and mortality for patients with acute respiratory distress syndrome in intensive care units in 50 countries. JAMA 2016:315:788-800

3. Definition Task Force ARDS, Ranieri VM, Rubenfeld GD, Thompson BT,

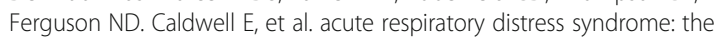
Berlin Definition. JAMA. 2012;307:2526-33.

4. Prescott HC, Calfee CS, Thompson BT, Angus DC, Liu VX. Toward smarter lumping and smarter splitting: rethinking strategies for Sepsis and acute respiratory distress syndrome clinical trial design. Am J Respir Crit Care Med. 2016;194:147-55.

5. Seymour CW, Kennedy JN, Wang S, Chang C-CH, Elliott CF, Xu Z, et al. Derivation, Validation, and Potential Treatment Implications of Novel Clinical Phenotypes for Sepsis. JAMA. 2019;321(20):2003-17.

6. Jabaudon M, Blondonnet R, Audard J, Fournet M, Godet T, Sapin V, et al. Recent directions in personalised acute respiratory distress syndrome medicine. Anaesth Crit Care Pain Med. 2018;37:251-8. 
7. Sinha P, Calfee CS. Phenotypes in acute respiratory distress syndrome: moving towards precision medicine. Curr Opin Crit Care. 2019;25:12-20.

8. Beitler JR, Goligher EC, Schmidt M, Spieth PM, Zanella A, Martin-Loeches I, et al. Personalized medicine for ARDS: the 2035 research agenda. Intensive Care Med. 2016:42:756-67.

9. Calfee CS, Delucchi K, Parsons PE, Thompson BT, Ware LB, Matthay MA, et al. Subphenotypes in acute respiratory distress syndrome: latent class analysis of data from two randomised controlled trials. Lancet Respir Med. 2014;2:611-20.

10. Bos LD, Schouten LR, van Vught LA, Wiewel MA, Ong DSY, Cremer O, et al. Identification and validation of distinct biological phenotypes in patients with acute respiratory distress syndrome by cluster analysis. Thorax. 2017;72:876-83.

11. Famous KR, Delucchi K, Ware LB, Kangelaris KN, Liu KD, Thompson BT, et al. Acute respiratory distress syndrome subphenotypes respond differently to randomized fluid management strategy. Am J Respir Crit Care Med. 2017;195:331-8.

12. Calfee CS, Delucchi KL, Sinha P, Matthay MA, Hackett J, Shankar-Hari M, et al. Acute respiratory distress syndrome subphenotypes and differential response to simvastatin: secondary analysis of a randomised controlled trial. Lancet Respir Med. 2018;6:691-8.

13. Matthay MA, Zemans RL, Zimmerman GA, Arabi YM, Beitler JR, Mercat A, et al. Acute respiratory distress syndrome. Nat Rev Dis Primers. 2019;5:18.

14. Matute-Bello G, Frevert CW, Martin TR. Animal models of acute lung injury. Am J Physiol Lung Cell Mol Physiol. 2008;295:L379-99.

15. Matute-Bello G, Downey G, Moore BB, Groshong SD, Matthay MA, Slutsky AS, et al. An official American Thoracic Society workshop report: features and measurements of experimental acute lung injury in animals. Am J Respir Cell Mol Biol. 2011;44:725-38.

16. Laffey JG, Kavanagh BP. Fifty years of research in ARDS. Insight into acute respiratory distress syndrome. From models to patients. Am J Respir Crit Care Med. 2017;196:18-28.

17. Rocco PRM, Nieman GF. ARDS: what experimental models have taught us. Intensive Care Med. 2016;42:806-10.

18. McAuley CDF, Hamid GF, Laffey UI, Abbott JG. McKenna J, et al. clinical grade allogeneic human mesenchymal stem cells restore alveolar fluid clearance in human lungs rejected for transplantation. Am J Physiol Lung Cell Mol Physiol. 2014;306(9):L809-15.

19. Park J, Kim S, Lim H, Liu A, Hu S, Lee J, et al. Therapeutic effects of human mesenchymal stem cell microvesicles in an ex vivo perfused human lung injured with severe E coli pneumonia. Thorax. 2019;74:43-50.

20. Lee JW, Fang X, Gupta N, Serikov V, Matthay MA. Allogeneic human mesenchymal stem cells for treatment of $\mathrm{E}$. coli endotoxin-induced acute lung injury in the ex vivo perfused human lung. Proc Natl Acad Sci U S A. 2009;106:16357-62.

21. Ross JT, Nesseler N, Lee J-W, Ware LB, Matthay MA. The ex vivo human lung: research value for translational science. JCI Insight. 2019;4(11).

22. Matthay MA, Calfee CS, Zhuo H, Thompson BT, Wilson JG, Levitt JE, et al. Treatment with allogeneic mesenchymal stromal cells for moderate to severe acute respiratory distress syndrome (START study): a randomised phase 2a safety trial. Lancet Respir Med. 2019;7:154-62.

23. Matthay MA, Goolaerts A, Howard JP, Lee JW. Mesenchymal stem cells for acute lung injury: preclinical evidence. Crit Care Med. 2010;38:S569-73.

24. Ware LB, Landeck M, Koyama T, Zhao Z, Singer J, Kern R, et al. A randomized trial of the effects of nebulized albuterol on pulmonary edema in brain-dead organ donors. Am J Transplant. 2014;14:621-8.

25. National Heart, Lung, and Blood Institute Acute Respiratory Distress Syndrome (ARDS) Clinical Trials Network, Matthay MA, Brower RG, Carson S, Douglas IS, Eisner M, et al. Randomized, placebo-controlled clinical trial of an aerosolized $\beta_{2}$-agonist for treatment of acute lung injury. Am J Respir Crit Care Med. 2011;184:561-8.

26. Blondonnet R, Audard J, Belville C, Clairefond G, Lutz J, Bouvier D, et al. RAGE inhibition reduces acute lung injury in mice. Sci Rep. 2017;7:7208.

27. Audard J, Godet T, Blondonnet R, Joffredo J-B, Paquette B, Belville C, et al. Inhibition of the receptor for advanced Glycation end-products in acute respiratory distress syndrome: a randomised laboratory trial in piglets. Sci Rep. 2019;9:9227.

28. Reddy K, O'Kane C, McAuley D. Corticosteroids in acute respiratory distress syndrome: a step forward, but more evidence is needed. Lancet Respir Med. 2020;8(20):30048-5. https://doi.org/10.1016/\$2213-2600(20)30048-5 [Epub ahead of print].

29. Camprubí-Rimblas M, Tantinyà N, Bringué J, Guillamat-Prats R, Artigas A. Anticoagulant therapy in acute respiratory distress syndrome. Ann Transl Med. 2018;6:36.
30. Jabaudon M, Boucher $P$, Imhoff E, Chabanne R, Faure J-S, Roszyk L, et al Sevoflurane for sedation in acute respiratory distress syndrome. A randomized controlled pilot study. Am J Respir Crit Care Med. 2017;195: 792-800.

31. McGowan J, Sampson M, Salzwedel DM, Cogo E, Foerster V, Lefebvre C. PRESS peer review of electronic search strategies: 2015 guideline statement. J Clin Epidemiol. 2016;75:40-6.

32. Hooijmans CR, Tillema A, Leenaars M, Ritskes-Hoitinga M. Enhancing search efficiency by means of a search filter for finding all studies on animal experimentation in PubMed. Lab Anim. 2010:44:170-5.

33. de Vries RBM, Hooijmans CR, Tillema A, Leenaars M, Ritskes-Hoitinga M. A search filter for increasing the retrieval of animal studies in Embase. Lab Anim. 2011:45:268-70.

34. Liberati A, Altman DG, Tetzlaff J, Mulrow C, Gøtzsche PC, loannidis JPA, et al The PRISMA statement for reporting systematic reviews and meta-analyses of studies that evaluate health care interventions: explanation and elaboration. J Clin Epidemiol. 2009;62:e1-34.

35. Harris PA, Taylor R, Thielke R, Payne J, Gonzalez N, Conde JG. Research electronic data capture (REDCap)--a metadata-driven methodology and workflow process for providing translational research informatics support. J Biomed Inform. 2009:42:377-81.

36. Higgins JPT, Altman DG, Gøtzsche PC, Jüni P, Moher D, Oxman AD, et al. The Cochrane Collaboration's tool for assessing risk of bias in randomised trials. BMJ. 2011;343:d5928.

37. Sterne JA, Hernán MA, Reeves BC, Savović J, Berkman ND, Viswanathan M, et al. ROBINS-l: a tool for assessing risk of bias in non-randomised studies of interventions. BMJ. 2016;355:14919.

38. Henderson VC, Kimmelman J, Fergusson D, Grimshaw JM, Hackam DG. Threats to validity in the design and conduct of preclinical efficacy studies: a systematic review of guidelines for in vivo animal experiments. PLoS Med. 2013;10:e1001489.

39. Kimmelman J, London AJ. Predicting harms and benefits in translational trials: ethics, evidence, and uncertainty. PLoS Med. 2011;8:e1001010.

40. Kilkenny C, Browne WJ, Cuthill IC, Emerson M, Altman DG. Improving bioscience research reporting: the ARRIVE guidelines for reporting animal research. PLoS Biol. 2010;8:e1000412.

41. Muthen LK, Muthen B. Mplus Version 8 User's Guide. Muthen \& Muthen; 2017.

42. DerSimonian R, Laird N. Meta-analysis in clinical trials revisited. Contemp Clin Trials. 2015:45:139-45.

43. Friedrich JO, Adhikari NKJ, Beyene J. The ratio of means method as an alternative to mean differences for analyzing continuous outcome variables in meta-analysis: a simulation study. BMC Med Res Methodol. 2008;8:32.

44. Chinn S. A simple method for converting an odds ratio to effect size for use in meta-analysis. Stat Med. 2000;19:3127-31.

45. Higgins JPT, Thompson SG. Quantifying heterogeneity in a meta-analysis. Stat Med. 2002;21:1539-58.

46. Meyer NJ, Calfee CS. Novel translational approaches to the search for precision therapies for acute respiratory distress syndrome. Lancet Respir Med. 2017:5:512-23.

47. Jabaudon M, Blondonnet R, Pereira B, Cartin-Ceba R, Lichtenstern C, Mauri T, et al. Plasma SRAGE is independently associated with increased mortality in ARDS: a meta-analysis of individual patient data. Intensive Care Med. 2018; 44:1388-99.

48. Sinha P, Delucchi KL, Thompson BT, McAuley DF, Matthay MA, Calfee CS, et al. Latent class analysis of ARDS subphenotypes: a secondary analysis of the statins for acutely injured lungs from sepsis (SAILS) study. Intensive Care Med. 2018;44:1859-69.

49. Bos LDJ, Scicluna BP, Ong DSY, Cremer O, van der Poll T, Schultz MJ. Understanding heterogeneity in biologic phenotypes of acute respiratory distress syndrome by leukocyte expression profiles. Am J Respir Crit Care Med. 2019;200:42-50.

50. Kitsios GD, Yang L, Manatakis DV, Nouraie M, Evankovich J, Bain W, et al. Host-response subphenotypes offer prognostic enrichment in patients with or at risk for acute respiratory distress syndrome. Crit Care Med. 2019;47: 1724-34.

51. Viswan A, Ghosh P, Gupta D, Azim A, Sinha N. Distinct metabolic Endotype mirroring acute respiratory distress syndrome (ARDS) subphenotype and its heterogeneous biology. Sci Rep. 2019;9:2108.

52. Sarma A, Calfee CS, Ware LB. Biomarkers and precision medicine: state of the art. Crit Care Clin. 2020;36:155-65. 
53. Seymour CW, Kerti SJ, Lewis AJ, Kennedy J, Brant E, Griepentrog JE, et al. Murine sepsis phenotypes and differential treatment effects in a

randomized trial of prompt antibiotics and fluids. Crit Care. 2019;23:384.

\section{Publisher's Note}

Springer Nature remains neutral with regard to jurisdictional claims in published maps and institutional affiliations.

Ready to submit your research? Choose BMC and benefit from:

- fast, convenient online submission

- thorough peer review by experienced researchers in your field

- rapid publication on acceptance

- support for research data, including large and complex data types

- gold Open Access which fosters wider collaboration and increased citations

- maximum visibility for your research: over $100 \mathrm{M}$ website views per year

At BMC, research is always in progress.

Learn more biomedcentral.com/submissions 